\title{
Implementation of GSM Based Water Meter A Step towards Automation in Billing System
}

\author{
Yogendra P Joshi ${ }^{1}$, M. B. Tadwalkar ${ }^{2}$ \\ ${ }^{I}$ (JSPM'S JSCOE, Hadpsar Pune) \\ 2(Asso.Prof. JSPM's JSCOE, Hadapsar Pune)
}

\begin{abstract}
Flow sensor based water meter presents very low cost, reliable, quick water meter system accompanying with existing GSM networks. Paddle wheel flow sensor JT121 measures the water flow accurately with the help of rotating paddles. Monthly water usage can be sent to municipal corporation office within fraction of seconds in the form of text message by using existing GSM network. Such metering system reduces manpower, with higher accuracy and less power consumption. It gives better results than any other metering systems such as mechanical, ultrasonic, electro-magnetic systems. By using this system water consumption can be observed in real time with controlled use of precious water resources. Water resources be managed for future planning. Non revenue water will be detected and loss can be avoided in distribution system. Keywords: flow sensor JT121, GSM network, GSM Mode, water management real time monitoring.
\end{abstract}

\section{Introduction}

Out of the earths surface almost $71 \%$ surface is covered with water. Only $3 \%$ of water can be used as drinking water or fresh water and $97 \%$ water is sea water which cannot be used by human out of $71 \%$ available water on earth. Tremendous population growth causes insufficient and uneven distribution of drinking water. So measuring the water usage and providing it with proper amount will limit the wastage of water in society. Previously some traditional methods were used such as mechanical meters in which water flow drives mechanical gears which drives moving arrow pointer on the water meter scale, such meter need to be read in customer premises which is time consuming process. Such mechanical meters overtaken by electronics meters which works on RF frequencies. RF transmitter is mounted on meter which will transmit water usage on monthly basis within a limited range which then collected by RF receiver based hand held device. Then by using computer interfacing billing can be done. Such system needs manpower and it takes some time in collecting and distributing bills.

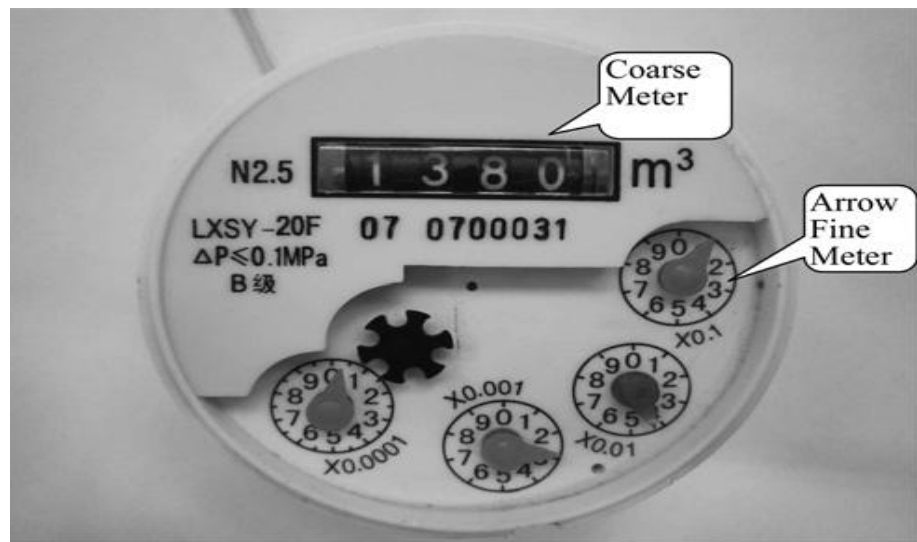

Fig.1 Mechanical Water Meter

\section{Existing System In Water Distribution System}

The Malkapur 24X7 water supply project is the first public sector initiative to have successfully provided water to the entire town. Launched in 2010, the scheme is implemented jointly by the Maharashtra Jeevan Pradhikaran and the Malkapur Nagar Panchayat. The scheme involves the use of a variety of innovative features. Firstly, it uses the design of the WaterGEMS software to design the system. Secondly, the use of High Density Polyethylene pipes that requires fewer joints makes for a more durable system as well as reduces the loss of water through leakages. Thirdly, the use of Automatic Meter Reading system has led to effective water auditing possible at any point of time and with cent per cent accuracy. Fourthly, the successful implementation of the telescopic tariff has meant that tariff recovery is linked to the volume transported over distances travelled. 
Uniformity has been created in the rate of water charges through the introduction of a volumetric tariff system. An automatic meter reading (AMR) system was introduced for monthly billing and recovery of water charges. The system also works to check excessive water usage by consumers. Computerized billing and customer friendly services such as SMS alerts have improved efficiency and generated higher revenues. Drive by system is used for AMR technology. The AMR meter generates an optical signal in each turn of the register dial. As shown in Fig.2 the unit collects pulse from the meter and stores the counting result in unit memory. In the present interval the dialog unit transmits the stored data to air, which is received on receiver driven near by it, which is then taken on computer by Bluetooth for immediate billing.

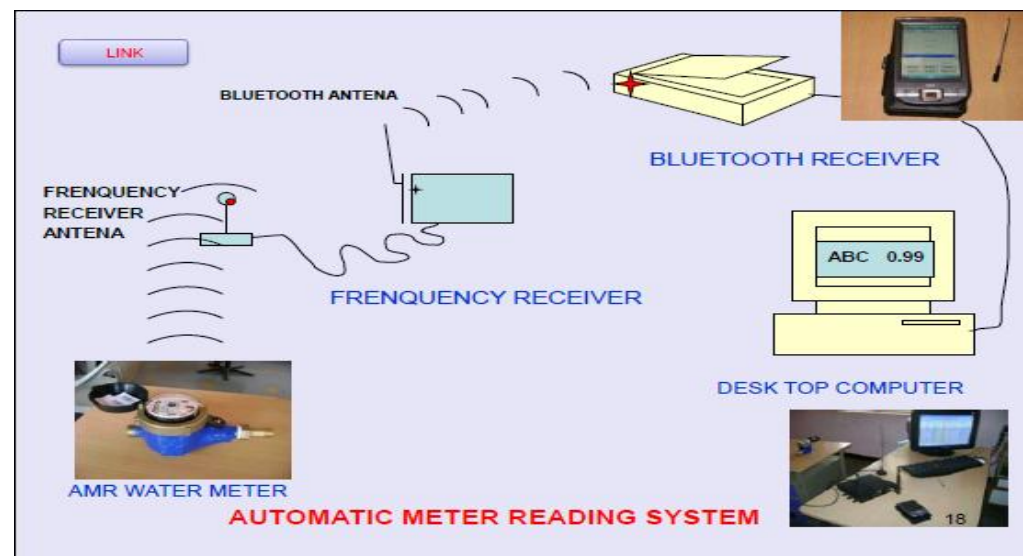

Fig.2 Automatic meter reading system

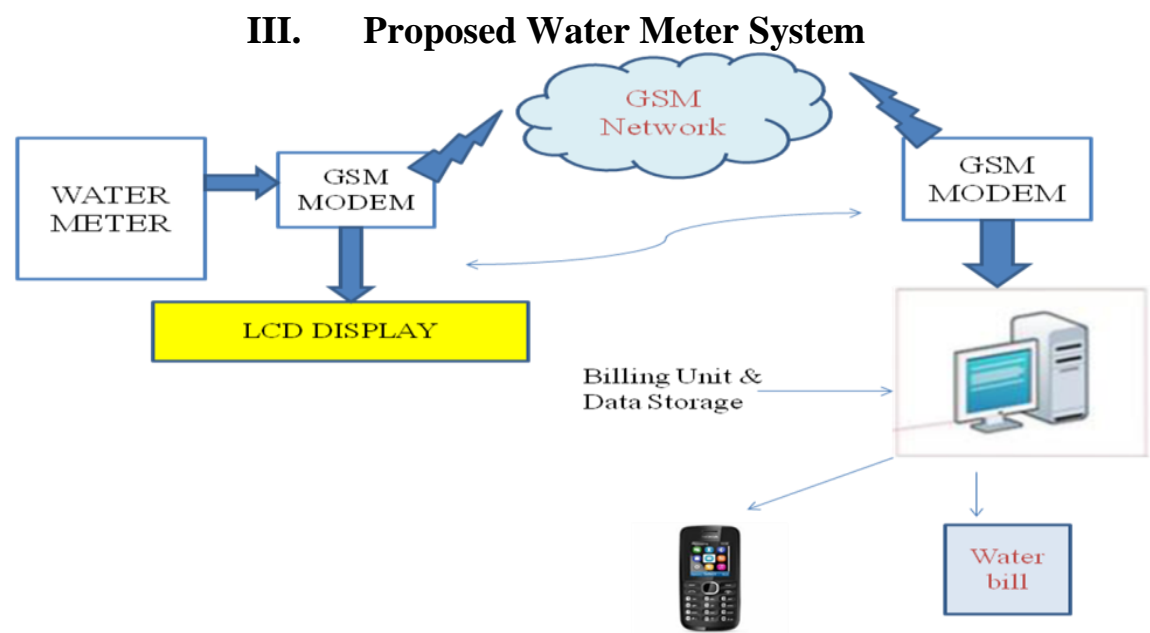

Fig.3 Proposed system block diagram

In above block diagram water meter block contains two blocks namely flow sensor JT-121 and micro controller. JT-121 interfaced with controller give water usage in terms of pulse count to GSM Modem. GSM modem receives water usage as a number string of numbers. It then transmitted to another GSM modem through existing GSM network. GSM modem is connected to computer through serial port, to give water usage. Billing software calculates bill based on amount of water used. Calculated bill can be sent through SMS, Email or in terms of print out to the customer. Whole mechanism of collection of water usage and providing bill take few minutes, which will be fastest and low cost process over the existing water distribution and billing system.

\section{Flow Sensor}

The VATS JT-121fig.4 flow sensor is a robust \& compact flow sensor, which generates frequency and signal proportional to the flow A ruggedly constructed range of paddle wheel flow sensors that is highly repeatable and delivers reliable performance. These paddle wheel flow sensors are powered from the meter that is driven by DC Voltage for output signal. Suitable for low pressure systems, these sensors measure the liquid flow rates in full pipes and are chemically compatible to many liquid process solutions. Set at proper insertion depth into the process flow. 


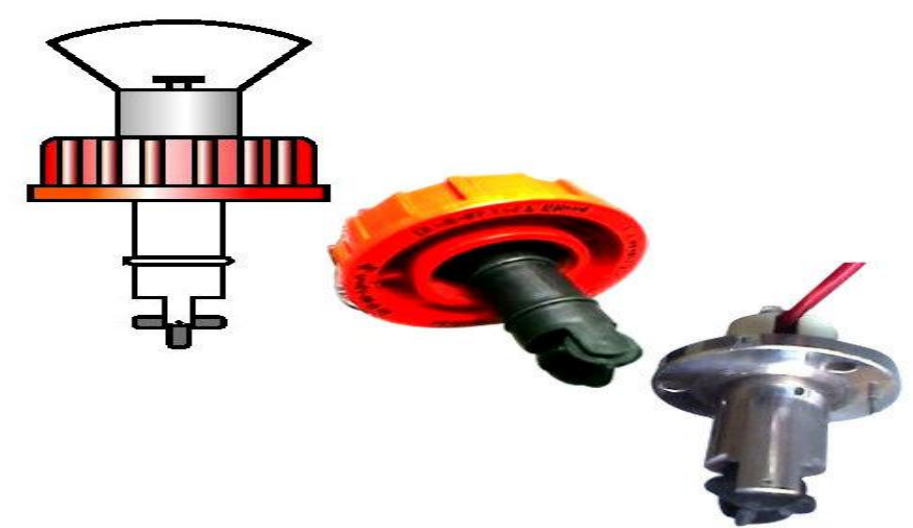

Fig.4. JT-121 Flow Sensor

The VATS JT-121 Flow Sensor gives output proportional to flow in square wave form, we can collect the output and can store in microcontroller. One of the port of micro controller will be configured as input port. Sensors output is connected to input port of controller, here at the falling edge of the wave controllers counter will be set till the wave ends. Meanwhile counter value will be incremented as per duration of the wave. At the end the value present in the timer will be given at output port by converting to decimal digits. We can make use of RTC for monthly billing (approximately 30 days).

\section{Gsm Modem}

Controller will give amount of water usage by user in the form of decimal values to the GSM Modem. GSM modem then formats the value in the Message form. By using AT commands. First we have to configure hyper terminal by giving it name. Now in properties we should select card phone option now clicking on OK button we are making it ready to use AT commands.

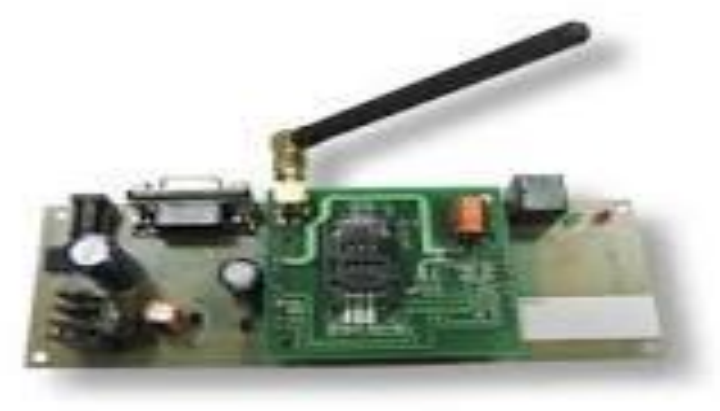

AT Commands for sending SMS

Fig.5.GSM Modem

1.Define message format by typing "AT+CMGF=1" (Text mode).

2.Set message center number by typing " $\mathrm{AT}+\mathrm{CSCA}=\mathrm{XXXXX"(Center} \mathrm{number} \mathrm{of} \mathrm{service} \mathrm{provider)}$

3.Enter recipient number by "AT+CMGS=YYYYYY".

4.Write message and press Ctrl-z.

\section{AT Commands for receiving SMS}

1.Define message format by typing "AT $+\mathrm{CMGF}=1$ "(Text mode).

2.Type “AT+CNMI=1,,2,0,0,0” In order to see all received messages.

In this project collection of water meter readings will be done by GSM network by SMS facility provided by GSM Technology .Numbers related with the water usage by user will be transmitted by the GSM modem at user side by sending just a SMS. This SMS will be received at the office end GSM modem. Hence no manpower is required, reading collection work is done automatically by GSM network which is already installed no need to make any special arrangement in GSM .

\section{Billing Unit}

Data of reading will be given to PC for billing purpose by GSM modem through DB9 connector. For billing we will be using GUI based on VB . In it total bill based on tariff ,taxes, discount will be displayed . We can print the same bill for circulation or may send E-mail or SMS . 


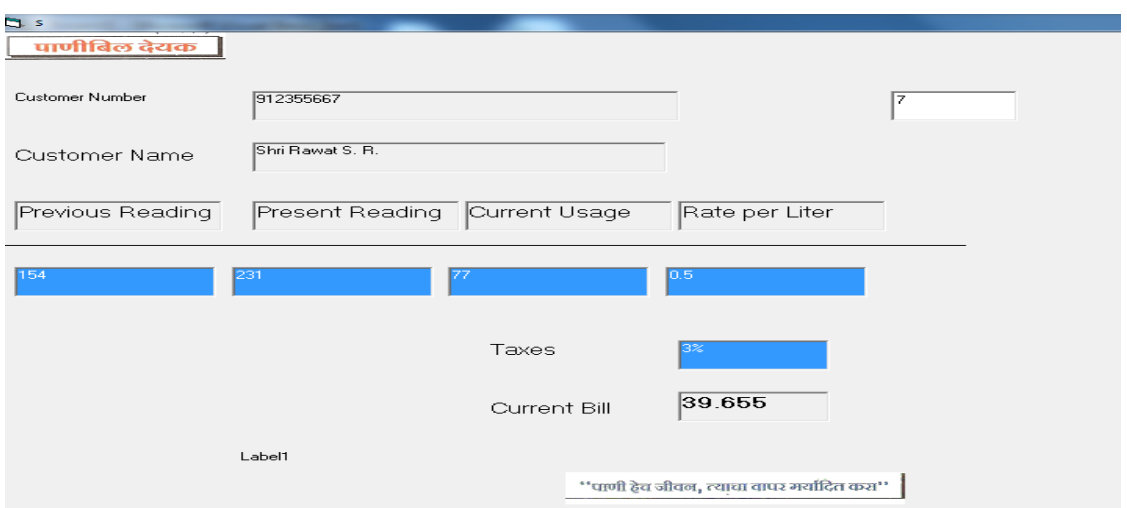

Fig.6 Billing window

\section{Water Leakage Detection}

Wireless sensor network setup for every zone- One cluster head and all other end user meter nodes in that zone as shown in fig 6.

Operating cluster head in two modes :

- Sending meter readings directly to central server for billing once in a month.

- Performing all computations at local cluster head and sending computed results to central server in order to alert for water leakage in that zone(once or twice in a day).

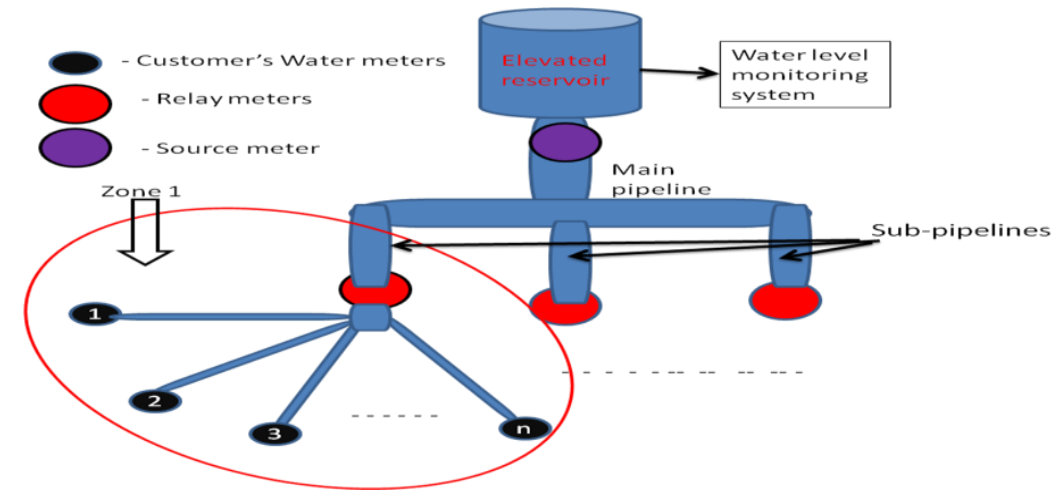

Fig.7 Water Leakage Detection System

\section{Conclusion}

Proposed system will provide accurate and real time water billing system. This overcomes existing systems in terms of cost and manpower required. Minimized cost of the whole system will support to use as economical and logical metering system. Monthly billing cycle can be maintained to limit the use of precious natural resource water.

\section{References}

[1] S.-C. Hsia, S.-W. Hsu, Y.-J. Chang,” Arrow-Pointer Sensor Design for Low-Cost Water Meter”. Published in IEEE SENSORS JOURNAL, VOL. 13, NO. 4, APRIL 2013

[2] Liu jie, Li hong-wei, Lv mou, Dong shen,” Investigation on Water Recording system for Large User of Water Supply Network Based on PLC". 1878-0296 @ 2011 Published by Elsevier Ltd. Selection and/or peer-review under responsibility of the Intelligent Information Technology Application Research Association. doi:10.1016/j.proenv.2011.12.155

[3] Aditi Dayal, Researcher's "Ensuring Efficient Water Supply" report on Malkapur's 24*7 water supply system. OneWorld.net February2012

[4] Maharashtra Jeevan Pradhikaran. .<www.mahamjp.com/newsite/awards/Malkapur_web_eng.pdf/

[5] Roy McKenzie and Brian Houston "Implementing Automatic Meter Reading for Water: A Reflective Approach to Weighing Options" FLORIDAWATER RESOURCES JOURNAL AUGUST 2011 